\title{
The effect on twinning rate of transferring double vitrified- warmed embryos in women of advanced reproductive age: a retrospective study
}

\author{
Yamei Xue $^{1,2}$, Kun Li $^{3}$, Songying Zhang ${ }^{\text {Corresp. 1,2 }}$ \\ 1 Assisted Reproduction Unit, Department of Obstetrics and Gynecology, Sir Run Run Shaw Hospital, School of Medicine, Zhejiang University, Hangzhou, \\ Zhejiang, China \\ ${ }^{2}$ Key Laboratory of Reproductive Dysfunction Management of Zhejiang Province, Hangzhou, China \\ 3 Department of Reproductive Physiology, Zhejiang Academy of Medical Sciences, Hangzhou, Zhejiang, China \\ Corresponding Author: Songying Zhang \\ Email address: zhangsongying@zju.edu.cn
}

Twin pregnancies are associated with greater risk of neonatal morbidity and mortality than a singleton. This study was performed to investigate the twin pregnancy rate when two vitrified-warmed embryos are transferred in women of advanced reproductive age ( $\geq 35$ years at the time of oocyte retrieval) and to evaluate the implications of findings in selecting candidates for elective single embryo transfer (eSET). A retrospective analysis of data which included 2038 women aged 35 to 45 years, who underwent vitrified-warmed double embryo transfer (DET), from January 2013 and December 2016 was undertaken. Pregnancy and twin rates were estimated after stratifying by prognostic profile. The twin pregnancy rate was lower in women with poor prognosis $(12 / 96,12.5 \%)$ as compared with that in women with favorable prognosis $(102 / 374,27.3 \%)$ and average prognosis (78/346, $22.5 \%)$ with significant differences $(P<.05)$. The twin rate for women with favorable prognosis were $29.2 \%$ (70/240) in the cycles of women aged $35-37$ years, $26.8 \%(26 / 97)$ in the cycles of women aged 38-40 years, and $16.2 \%$ (6/37) in the cycles of women $>40$ years. The twin rate for women with average prognosis were $25.8 \%(51 / 198)$ in the cycles of women aged 35-37 years, $22.0 \%(22 / 100)$ in the cycles of women aged 38-40 years, and $10.4 \%$ (5/48) in the cycles of women $>40$ years. The twin rate for women with poor prognosis were $15.3 \%(9 / 59)$ in the cycles of women aged $35-37$ years, $10.3 \%(3 / 29)$ in the cycles of women aged 38-40 years, and $0 \%(0 / 8)$ in the cycles of women $>40$ years. From these results, it was concluded that women with a favorable or average prognosis have a high risk of twin pregnancies. The finding can be used to guide future practice: i.e. performing ESET in women with favorable or average prognosis and DET in women with poor prognosis. 


\section{The effect on twinning rate of transferring double}

\section{2 vitrified-warmed embryos in women of advanced}

\section{3 reproductive age: a retrospective study}

4

5 Yamei Xue ${ }^{1,2}$, Kun $\mathrm{Li}^{3}$, Songying Zhang ${ }^{1,2 *}$

6

$7{ }^{1}$ Assisted Reproduction Unit, Department of Obstetrics and Gynecology, Sir Run Run Shaw

8 Hospital, School of Medicine, Zhejiang University, Hangzhou, Zhejiang, China

$9{ }^{2}$ Key Laboratory of Reproductive Dysfunction Management of Zhejiang Province, Hangzhou, 10 China

$11{ }^{3}$ Department of Reproductive Physiology, Zhejiang Academy of Medical Sciences, Hangzhou, 12 Zhejiang, China

13

14 Corresponding Author:

15 Songying Zhang ${ }^{1,2}$

16

17

18

19

20

21

22

23

24

25

26

27

28

29
No.3 Qingchun East Road, Hangzhou, Zhejiang, 310016, China

Email: zhangsongying@zju.edu.cn

\section{Abstract}

1 Twin pregnancies are associated with greater risk of neonatal morbidity and mortality than a singleton. This study was performed to investigate the twin pregnancy rate when two vitrified-

warmed embryos are transferred in women of advanced reproductive age $(\geqslant 35$ years at the time 4 of oocyte retrieval) and to evaluate the implications of findings in selecting candidates for 25 elective single embryo transfer (eSET). A retrospective analysis of data which included 2038 6 women aged 35 to 45 years, who underwent vitrified-warmed double embryo transfer (DET), 7 from January 2013 and December 2016 was undertaken. Pregnancy and twin rates were 28 estimated after stratifying by prognostic profile. The twin pregnancy rate was lower in women 29 with poor prognosis $(12 / 96,12.5 \%)$ as compared with that in women with favorable prognosis 
$30(102 / 374,27.3 \%)$ and average prognosis $(78 / 346,22.5 \%)$ with significant differences $(P<.05)$.

31 The twin rate for women with favorable prognosis were 29.2\% (70/240) in the cycles of women

32

33

34

35

36

37

38

39

40

41

\section{Introduction}

43

44

45

46

47

48

49

50

51

52

53

54

55

56

57

58

59

60 aged 35-37 years, 26.8\% (26/97) in the cycles of women aged 38-40 years, and 16.2\% (6/37) in the cycles of women $>40$ years. The twin rate for women with average prognosis were $25.8 \%$ $(51 / 198)$ in the cycles of women aged 35-37 years, $22.0 \%(22 / 100)$ in the cycles of women aged $38-40$ years, and $10.4 \%$ (5/48) in the cycles of women $>40$ years. The twin rate for women with poor prognosis were $15.3 \%$ (9/59) in the cycles of women aged $35-37$ years, $10.3 \%(3 / 29)$ in the cycles of women aged 38-40 years, and $0 \%(0 / 8)$ in the cycles of women $>40$ years. From these results, it was concluded that women with a favorable or average prognosis have a high risk of twin pregnancies. The finding can be used to guide future practice: i.e. performing eSET in women with favorable or average prognosis and DET in women with poor prognosis.

Since the widespread introduction of assisted reproductive techniques (ART) in the last decades, the transfer of more than one embryo with the aim of maximizing pregnancy rates has led to an increase in the rate of twin pregnancies (Vlachadis et al. 2014). Twin gestations are more likely to have adverse maternal and neonatal outcomes compared to singletons, including gestational hypertension, premature births, intrauterine growth retardation, and low birth weight (Saccone et al. 2019; Young \& Wylie 2012). Twin gestation also compound the health threats to women with advanced age, who have a three-time higher risk of pregnancy-related mortality than do younger counterparts (MacKay et al. 2001). Ideally, the goal of ART is to achieve a singleton gestation (Styer et al. 2016; Thurin et al. 2004).

2 Elective single embryo transfer (eSET) is encouraged, as is the subsequent reduction in twin 3 pregnancies. Age is a primary clinic criterion when considering a woman's candidacy for eSET.

4 Martin et al. specified the strongest negative predictor of double embryo implantation was age

$5 \geqslant 35$ years compared to age $<35$ years after double embryo transfer (DET) (Martin et al. 2016).

6 Myo sun kim et al. reported maternal age $\leqslant 35$ years was the cut-off value to predict twin 7 pregnancy following DET (Kim et al. 2015). Therefore, recommendations for eSET have 8 focused mainly on women aged < 35 years who have a favorable prognosis (Thurin et al. 2004). 59 Increasing age is associated with a lower chance of multiple pregnancies (Martin et al. 2016). 60 For women with over 35 years of age, transferring two embryos is still their first choice, even 
61 with a favorable prognosis, in order to maximize the chance of pregnancy with a single

62 treatment. However, according to a large study performed in the USA, women of over 35 years

63 of age had up to $22.1 \%$ of double embryo implantation in the favorable prognosis group in DET

64 cycles (Martin et al. 2016). A report from Finland estimated the twin pregnancy rate to be 17.7\%,

65 when two embryos were transferred in women aged 36-39 years (Veleva et al. 2006). In the case

66 that two embryos are transferred and both are implanted, there may be a missed chance for a

67 successful eSET.

68 In China, the eSET policy has gradually been well accepted by women aged $\leqslant 35$ years with a

69 good prognosis without provision of law, and practice has led to a decline in the number of twin 70 pregnancy after IVF (Yang et al. 2018). However, for women older than 35 years, data from existing studies are limited and have not been able to provide robust evidence on DET or eSET.

72 The present study was therefore carried out to evaluate the outcome of DET in older Chinese

73

74 75

76

\section{Materials \& Methods}

\section{Subjects}

79

80

81

82

83

84

85

86

87

88

89

In early 2012, a decision was made at our center to limit patients to the transfer of a maximum of two embryos, to control for a multiple pregnancy rate. This retrospective study analyzed the data of 2038 women $\geqslant 35$ years at the time of oocyte retrieval, who underwent transfer of vitrifiedwarmed double embryos at the Reproductive Medicine Center, Sir Run Run Shaw Hospital, China between January 2013 and December 2016. Each patient contributed only one cycle. All of the transferred embryos were at the Day 3 stage. Exclusion criteria included cycles involving mixed fresh/thawed embryo transfer, oocyte donation and those in which embryos were cryopreserved by slow freezing. Reproductive Medical Ethics Committee of Sir Run Run Shaw Hospital, College of Medicine, Zhejiang University granted Ethical approval to carry out the study within its facilities (Ethical Application Ref: SRRSHRMEC2017005). Written informed consent was obtained from each participating couple. 
90 The prognostic profiles (i.e. favorable/average/poor) were based on two important predictive 91 factors for pregnancy with IVF: number of previous cycles and embryo quality (Practice 92 Committee of the American Society for Reproductive Medicine, Practice Committee of the 93 Society for Assisted Reproductive Technology 2009). Since maternal age is the most important 94 factor, we divide age into the three groups (35-37, 38-40 and $>40$ years), to further evaluate the 95 role that maternal age played in twin pregnancy rates. The definition of favorable, average and 96 poor prognosis was shown in Table 1. The favorable prognosis population was defined as 97 women aged $\geqslant 35$ years who (1) underwent their first cycle of embryo transfer and had two 98 good-quality embryos transferred, (2) had a previous successful embryo transfer and $\geqslant 1$ goodquality embryos transferred. The average prognosis population was defined as women who aged $\geqslant 35$ years who (1) did not have two good-quality embryos transferred in the first cycle,(2) failed to get pregnant in their first transfer cycle and had at least one good-quality embryos transferred, or (3) had a previous successful embryo transfer and no good-quality embryos transferred.

103

104 105

106

107 108

109

110

111

112

113

114

115

116

117

118

119

Women with a poor prognosis were women aged $\geqslant 35$ years who had $\geqslant 1$ failed embryo transfer and no good-quality embryos transferred.

\section{IVF procedure}

The ovarian stimulation and IVF procedures have been previously described (Xue et al. 2014; Xue et al. 2018). Briefly, embryo culture with sequential media was carried out as follows: fertilization was performed in fertilization medium (G-IVF, Vitrolife Sweden AB, Sweden) containing 10\% serum substitute supplement (SSS, Irvine Scientific, Santa Ana, CA). The following morning, the oocytes were individually placed in microdrops in cleavage medium (G-1, Vitrolife Sweden AB, Sweden) with $12 \% \mathrm{SSS}$ and incubated in incubators at $37^{\circ} \mathrm{C}$ with $6 \%$ $\mathrm{CO}_{2}, 5 \% \mathrm{O}_{2}$ and $89 \% \mathrm{~N}_{2}$ for $48 \mathrm{~h}$ before being frozen. Morphological assessment of embryos was based on the number of blastomeres and the proportion of cytoplasmic fragments and the uniformity of blastomeres (Xue et al. 2014).

\section{Vitrification and warming protocols}

Vitrification-warming procedures were performed using the Cryotop cryopreservation technologies (Kitazato, BioPharma Co. Ltd., Fuji City, Japan). The thawed embryos cultured in 
120 G-2 medium containing 12\% SSS for $2 \mathrm{~h}$ prior to transfer. Embryos were defined as surviving if

121 they had with $\geqslant 50 \%$ of intact blastomeres immediately after warming. The presence of damaged

122 blastomeres was recorded after warming.

123

124 Endometrial preparation

125 Warming embryos were transferred in natural or hormone replacement treatment (HRT) cycles 126 as described previously (Xue et al. 2018). For women with regular menstrual cycles, warmed 127 embryos were transferred in natural cycles. For women with irregular ovulation or anovulatory 128 cycles, HRT was used. Luteal phase support was provided with intramuscular injections of 129 progesterone 80mg (Xianju Pharmaceutical factory, China) from 3 days before ET until a viable 130 intrauterine pregnancy on ultrasound examination was observed.

131

132 Outcomes

133 For each transfer, this study observed whether or not DET resulted in a clinical pregnancy and, if 134 so, in a singleton or a dizygotic twinning. Clinical pregnancy was defined by the observation of a 135 gestational sac with or without fetal heartbeat on ultrasound evaluation 35 days after embryo 136 transfer. The number of sacs was taken as the number of successful implantations. Live birth 137 referred to the birth of one or more live infants regardless of the duration of pregnancy.

\section{Statistical analysis}

140 For the statistical analyses, the statistical program Statistical Package for Social Sciences 141 version 20.0 for Windows (SPSS, Chicago, IL, USA) was used. The distributions of continuous

142 variables were evaluated by Kolmogorov Smirnov test. The variables were compared with one 143 way analysis of variance (ANOVA) test and the nonparametric Kruskal-Wallis test depending on 144 normal or abnormal distribution, respectively. The continuous variables were presented as mean $145 \pm$ standard deviation (SD). Categorical variables were presented as $\mathrm{n}(\%)$ and compared with the 146 Pearson's Chi-squared or Fisher exact test on the basis of sample size. Post hoc pairwise 147 comparisons were performed using the Bonferroni correction. The result was considered 148 significant if the $P$-value was $<0.05$. 
151 During the study period, 2038 women at least 35 years old underwent vitrified-warmed DET.

152 They were divided according to prognosis to 812 cycles performed on women with favorable 153 prognosis, 906 cycles performed on women with average prognosis and 320 cycles performed on 154 women with poor prognosis.

155 The patients' characteristics are summarized for each prognostic category in Table 2. The 156 most common infertility cause was male factor infertility for patients with favorable and poor 157 prognosis and tubal pathology infertility for women with average prognosis. The differences for 158 patients' age, fertilization method, and number of good-quality embryos transferred among the 159 three groups were statistically significant, but the maternal BMI, type of infertility, length of 160 embryo cryopreservation, damaged blastomere, embryo survival rate, endometrial thickness, and 161 endometrial preparation were similar among the three groups of patients.

162 In the cycles of women with a favorable prognosis, the clinical pregnancy rate was $46.1 \%$ $163(374 / 812)$ per transfer with twin pregnancy rate of $27.3 \%(102 / 374)$ and the live birth rate of $41.3 \%$ 164 (335/812).In the cycles of women with an average prognosis, the clinical pregnancy rate was $16538.2 \%$ (346/906), the twin pregnancy rate was 22.5\% (78/346) and the live birth rate was 35.1\% 166 (318/906).The rates of clinical pregnancy, twin pregnancy and live birth for women with poor 167 prognosis were $30.0 \%(96 / 320), 12.5 \%(12 / 96)$ and $25.3 \%(81 / 320)$, respectively. The 168 differences for clinical pregnancy, twin and live birth rates among the three groups were 169 statistically significant $(P<.01)$.

170 The patients were subdivided by the women's age into three groups: $35-37,38-40$ and $>40$ 171 years. The pregnancy, twin and live birth rates for women with favorable prognosis were $49.3 \%$ 172 (240/487), 29.2\% (70/240) and 44.6\% (217/487) in the cycles of women aged 35-37 years, $17343.9 \%(97 / 221), 26.8 \%$ (26/97) and 38.9\% (86/221) in the cycles of women aged 38-40 years, $17435.6 \%(37 / 104), 16.2 \%(6 / 37)$ and $30.8 \%(32 / 104)$ in the cycles of women $>40$ years. The 175 pregnancy, twin and live birth rates for women with average prognosis were 41.0\% (198/483), $17625.8 \%(51 / 198)$ and $38.3 \%$ (185/483) in the cycles of women aged 35-37 years, 38.2\% (100/262), $17722.0 \%(22 / 100)$ and $34.7 \%(91 / 262)$ in the cycles of women aged 38-40 years, 29.8\% (48/161), $17810.4 \%(5 / 48)$ and $26.1 \%(42 / 161)$ in the cycles of women $>40$ years. The pregnancy, twin and 179 live birth rates for women with poor prognosis were $36.0 \%(59 / 164), 15.3 \%(9 / 59)$ and $31.1 \%$ $180(51 / 164)$ in the cycles of women aged 35-37 years, 29.6\% (29/98), 10.3\% (3/29) and 24.5\% 
181 (24/98) in the cycles of women aged 38-40 years, $13.8 \%(8 / 58), 0 \%(0 / 8)$ and $10.3 \%(6 / 58)$ in 182 the cycles of women $>40$ years. The data are reported in Table 3.

\section{Discussion}

185 The relaxation of the one child policy in 2013 in China and introduction of the two child policy 186 in 2015 has led to more and more women of advanced reproductive age seeking assisted 187 reproduction (Liang et al. 2018). One of the major unsolved issues in IVF is optimizing 188 pregnancy rates while limiting multiple pregnancy rates in older women $(\geqslant 35$ years) with 189 favorable or average prognosis, such as women who have one or more good-quality embryos available in the first transfer cycle. This retrospective evaluation of DET based on the prognostic profile of the individual patient shows that in women with favorable and average prognosis DET results in good pregnancy rates, but at the expense of high twin pregnancy rates. Women with a poor prognosis undergoing DET have reasonable clinical pregnancy rate with a limited twin pregnancy rate $(12.5 \%)$.

It is well known that maternal age is the most significant indicator for double embryo implantation following DET (Kim et al. 2015; Martin et al. 2016). Various studies have consistently shown that oocytes from women over 40 years of age have decreased quality (Alasmari et al. 2016; Hourvitz et al. 2009); subsequently, the resultant embryos will show declining developmental potentials (Marquez et al. 2000). A large prospective study concerning the effect of age on perinatal livebirth outcomes varied by the number of embryo transferred showed that the risk of multiple births was lower among women older than 40 years (Lawlor \& 202 Nelson 2012). Similar to previous studies, the data in this study suggests that women older than 40 years old have a reduced risk of double embryo implantation when using their own eggs, irrelevant of the prognosis. Thus transfer of two embryos is applicable to women older than 40 years. However, in women aged 35-40 years with favorable and average prognosis, clinical pregnancy rates were good, but twin pregnancy rates were high enough to reach more than $20 \%$, which is slightly higher than previously reported in women aged 36-39 years (16.6\%) (Veleva et al. 2006). It is clearly that in these women better selection is obviously necessary. Given that women at these age groups has an increased perinatal risk and may become particularly vulnerable to multiple pregnancy (Davis et al. 2008; Ubaldi et al. 2015), possible eSET should be 
211 offered to women aged 35-40 years with favorable and average prognosis to increase the safety

212 of ART; however, further data will be needed to support this conclusion.

213 Vitrification, or the rapid-freezing of embryos, has been implemented in many IVF centers

214 and has been shown in comparative trials to be associated with higher rates of post-thaw survival

215 and intact embryos than slow-freezing method (Debrock et al. 2015). The risk for cycle

216 cancellation due to cryopreservation damage was becoming extremely low. Several studies have

217 shown predictors of implantation after transfer of slow-freezing embryos including: percent

218 blastomere survival after thaw (El-Toukhy et al. 2003; Kaser et al. 2013) and survival rate of

219 intact embryo (Burns et al. 1999; El-Toukhy et al. 2003). In the present study, the use of

220 vitrification technology has resulted in embryo survival rate of $97.8 \%$ and intact embryo rate of

$22189.2 \%$. The factors reported in previous studies such as survival rate of embryos and percent

222 blastomere survival after thaw may not be related to embryo implantation in this study. Thus,

223 vitrification may emerge as a method to optimize the safety and efficacy of assisted

224 reproduction.

225 An important question remains: what are acceptable twin pregnancy rates in Chinese women 226 older than 35 years? Since introduction of the two child policy, most couples desire more than 227 one child ( $\mathrm{Li} \&$ Deng 2017). For women 35 years of age and older, a twin pregnancy may be the 228 only chance to conceive and deliver a second child. It has been shown in the literatures that a 229 significant proportion of women with advanced age have a clear preference for twins and view a 230 twin pregnancy as being more desirable than having no child, even if associated with adverse 231 maternal outcomes (Fiddelers et al. 2011; van Loendersloot et al. 2013). So, the clinical decision 232 between eSET and DET should not be based solely on the characteristics of the treatment cycle, 233 but also on the preference of the patients.

234 The study has several limitations. First, due to the retrospective nature of the study and non235 specific criteria for DET, there is a possibility of a selection bias. Also, this study applies to an 236 unselected group of patients with aged $\geqslant 35$ years, namely, those with vitrification and transfer 237 of two cleavage stage embryos. Because of the limited data, it was not possible to distinguish 238 between those patients who had DET due to clinical history and those who had DET because 239 only two embryos were suitable for transfer. Additionally, in early 2012, in order to reduce the 240 multiple pregnancy rates, we implemented a policy of the transfer of a maximum of two 241 embryos. The 2013 ASRM guidelines (Practice Committee of American Society for 
242 Reproductive Medicine, Practice Committee of Society for Assisted Reproductive 2013)

243 recommended limits on the number of embryos transferring as two cleavage-stage embryos in

244 favorable prognosis and three embryos in all others. The recent ASRM statement (Practice

245 Committee of the American Society for Reproductive Medicine, Practice Committee of the

246 Society for Assisted Reproductive Technology 2017) was published in 2017 after our study had

247 been terminated. Even one year prior to the 2013 ASRM recommendations, our policy was to

248 reduce the multiple pregnancy rates in this study. Moreover, our criteria defined the prognostic

249 profiles were in line with the ASRM (patient age, embryo quality and previous IVF cycle).

250 Finally, the quality of embryos transferred was only assessed by morphological score, which is

251 believed to a simple, less costly and generally accepted method, but may not be consistent with

252 true embryo quality (Sigalos et al. 2016). However, an important strength that enhances the

253 validity of our data is the consistency of our clinical and laboratory protocols during the four-

254 year study period (e.g. endometrial preparation, ultrasound-guided embryo transfer, the protocols

255 for embryo grading, embryo cryopreservation and thaw protocols).

256

\section{Conclusions}

258 From these results, it was concluded that women with a favorable or average prognosis have a

259 high risk of twin pregnancies. The finding can be used to guide future practice: i.e. performing

260 eSET in women with favorable or average prognosis and DET in women with a poor prognosis.

261 We hope that these results will encourage physicians to better tailor patient older than 35 years or

262 counsel patients on eSET, which reduce multiple pregnancy and associated complications. The

263 novel aspect of our study is that we stratified our comparisons by prognosis, which may be

264 useful in making decisions between eSET and DET for women of advanced reproductive age.

265

266 Funding

267 This study was supported by Natural Science Foundation of Zhejiang Province (No. LY17H040002), the

268 Sci\&Tech Program Project of Zhejiang Province (2018C37126), the Health Sci\&Tech Plan Project of

269 Zhejiang Province (2019KY363), the Special Supporting Program Project for the Research Institutions of

270 Zhejiang Province (C11920D-04) and the Zhejiang Provincial Program for the Cultivation of High-level

271 Innovative Health talents.

272 
273 Author Contributions Yamei Xue: Study idea and design, data management and analysis, manuscript

274 writing and editing. Kun Li: analysis of data and editing of the manuscript. Songying Zhang: Study idea

275 and design, data management and analysis, editing of the manuscript. All authors read and approved the

276 final manuscript.

277

278 Conflict of interest All authors declare that they have no conflicts of interest to this work.

279

\section{References}

281

282

283

284

285

286

287

288

289

290

291

292

293

294

295

296

297

298

299

300

301

302

303

304

305

306

307

308

309

310

311

312

313

314

315

316

317

318

319
Alasmari NM, Son WY, and Dahan MH. 2016. The effect on pregnancy and multiples of transferring 1-3 embryos in women at least 40 years old. J Assist Reprod Genet 33:1195-1202. 10.1007/s10815-016-0749-6

Burns WN, Gaudet TW, Martin MB, Leal YR, Schoen H, Eddy CA, and Schenken RS. 1999. Survival of cryopreservation and thawing with all blastomeres intact identifies multicell embryos with superior frozen embryo transfer outcome. Fertil Steril 72:527-532.

Davis LB, Lathi RB, Westphal LM, and Milki AA. 2008. Elective single blastocyst transfer in women older than 35. Fertil Steril 89:230-231. 10.1016/j.fertnstert.2007.02.047

Debrock S, Peeraer K, Fernandez Gallardo E, De Neubourg D, Spiessens C, and D'Hooghe TM. 2015. Vitrification of cleavage stage day 3 embryos results in higher live birth rates than conventional slow freezing: a RCT. Hum Reprod 30:1820-1830. 10.1093/humrep/dev134

El-Toukhy T, Khalaf Y, Al-Darazi K, Andritsos V, Taylor A, and Braude P. 2003. Effect of blastomere loss on the outcome of frozen embryo replacement cycles. Fertil Steril 79:1106-1111.

Fiddelers AA, Nieman FH, Dumoulin JC, van Montfoort AP, Land JA, Evers JL, Severens JL, and Dirksen CD. 2011. During IVF treatment patient preference shifts from singletons towards twins but only a few patients show an actual reversal of preference. Hum Reprod 26:2092-2100. 10.1093/humrep/der127

Hourvitz A, Machtinger R, Maman E, Baum M, Dor J, and Levron J. 2009. Assisted reproduction in women over 40 years of age: how old is too old? Reprod Biomed Online 19:599-603.

Kaser DJ, Missmer SA, Correia KF, Ceyhan ST, Hornstein MD, and Racowsky C. 2013. Predictors of twin live birth following cryopreserved double embryo transfer on day 3. J Assist Reprod Genet 30:1023-1030. 10.1007/s10815-013-0039-5

Kim MS, Kim JH, Jee BC, Suh CS, and Kim SH. 2015. Factors affecting occurrence of twin pregnancy after double embryo transfer on day 3. J Obstet Gynaecol Res 41:1223-1228. 10.1111/jog.12687

Lawlor DA, and Nelson SM. 2012. Effect of age on decisions about the numbers of embryos to transfer in assisted conception: a prospective study. Lancet 379:521-527. 10.1016/S0140-6736(11)61267-1

Li Q, and Deng D. 2017. New medical risks affecting obstetrics after implementation of the two-child policy in China. Front Med 11:570-575. 10.1007/s11684-017-0552-5

Liang J, Mu Y, Li X, Tang W, Wang Y, Liu Z, Huang X, Scherpbier RW, Guo S, Li M, Dai L, Deng K, Deng C, Li Q, Kang L, Zhu J, and Ronsmans C. 2018. Relaxation of the one child policy and trends in caesarean section rates and birth outcomes in China between 2012 and 2016: observational study of nearly seven million health facility births. BMJ 360:k817. 10.1136/bmj.k817

Mackay AP, Berg CJ, and Atrash HK. 2001. Pregnancy-related mortality from preeclampsia and eclampsia. Obstet Gynecol 97:533-538.

Marquez C, Sandalinas M, Bahce M, Alikani M, and Munne S. 2000. Chromosome abnormalities in 1255 cleavagestage human embryos. Reprod Biomed Online 1:17-26.

Martin C, Chang J, Boulet S, Jamieson DJ, and Kissin D. 2016. Factors predicting double embryo implantation following double embryo transfer in assisted reproductive technology: implications for elective single embryo transfer. J Assist Reprod Genet 33:1343-1353. 10.1007/s10815-016-0770-9

Practice Committee of American Society for Reproductive Medicine, Practice Committee of Society for Assisted Reproductive T. 2013. Criteria for number of embryos to transfer: a committee opinion. Fertil Steril 99:44- 
46. 10.1016/j.fertnstert.2012.09.038

Practice Committee of the American Society for Reproductive Medicine, Practice Committee of the Society for Assisted Reproductive Technology. 2009. Guidelines on number of embryos transferred. Fertil Steril 92:1518-1519. 10.1016/j.fertnstert.2009.08.059

Practice Committee of the American Society for Reproductive Medicine, Practice Committee of the Society for Assisted Reproductive Technology. 2017. Guidance on the limits to the number of embryos to transfer: a committee opinion. Fertil Steril 107:901-903. 10.1016/j.fertnstert.2017.02.107

Saccone G, Zullo F, Roman A, Ward A, Maruotti G, Martinelli P, and Berghella V. 2019. Risk of spontaneous preterm birth in IVF-conceived twin pregnancies. J Matern Fetal Neonatal Med 32:369-376. 10.1080/14767058.2017.1378339

Sigalos G, Triantafyllidou O, and Vlahos NF. 2016. Novel embryo selection techniques to increase embryo implantation in IVF attempts. Arch Gynecol Obstet 294:1117-1124. 10.1007/s00404-016-4196-5

Styer AK, Luke B, Vitek W, Christianson MS, Baker VL, Christy AY, and Polotsky AJ. 2016. Factors associated with the use of elective single-embryo transfer and pregnancy outcomes in the United States, 2004-2012. Fertil Steril 106:80-89. 10.1016/j.fertnstert.2016.02.034

Thurin A, Hausken J, Hillensjo T, Jablonowska B, Pinborg A, Strandell A, and Bergh C. 2004. Elective single-embryo transfer versus double-embryo transfer in in vitro fertilization. N Engl J Med 351:2392-2402. 10.1056/NEJMoa041032

Ubaldi FM, Capalbo A, Colamaria S, Ferrero S, Maggiulli R, Vajta G, Sapienza F, Cimadomo D, Giuliani M, Gravotta E, Vaiarelli A, and Rienzi L. 2015. Reduction of multiple pregnancies in the advanced maternal age population after implementation of an elective single embryo transfer policy coupled with enhanced embryo selection: pre- and post-intervention study. Hum Reprod 30:2097-2106. 10.1093/humrep/dev159

van Loendersloot L, van Wely M, Goddijn M, Repping S, Bossuyt P, and van der Veen F. 2013. Pregnancy and twinning rates using a tailored embryo transfer policy. Reprod Biomed Online 26:462-469. 10.1016/j.rbmo.2013.01.010

Veleva Z, Vilska S, Hyden-Granskog C, Tiitinen A, Tapanainen JS, and Martikainen H. 2006. Elective single embryo transfer in women aged 36-39 years. Hum Reprod 21:2098-2102. 10.1093/humrep/del137

Vlachadis N, Vrachnis N, and Economou E. 2014. Fertility treatments and multiple births in the United States. N Eng/ J Med 370:1069-1070. 10.1056/NEJMc1400242

Xue Y, Tong X, Jiang L, Zhu H, Yang L, and Zhang S. 2014. Effect of vitrification versus slow freezing of human day 3 embryos on beta-hCG levels. J Assist Reprod Genet 31:1037-1043. 10.1007/s10815-014-0259-3

Xue Y, Tong X, Zhu H, Li K, and Zhang S. 2018. Freeze-all embryo strategy in poor ovarian responders undergoing ovarian stimulation for in vitro fertilization. Gynecol Endocrinol 34:680-683. 10.1080/09513590.2018.1427715

Yang L, Cai S, Zhang S, Kong X, Gu Y, Lu C, Dai J, Gong F, Lu G, and Lin G. 2018. Single embryo transfer by Day 3 time-lapse selection versus Day 5 conventional morphological selection: a randomized, open-label, noninferiority trial. Hum Reprod 33:869-876. 10.1093/humrep/dey047

Young BC, and Wylie BJ. 2012. Effects of twin gestation on maternal morbidity. Semin Perinatol 36:162-168. 10.1053/j.semperi.2012.02.007 


\section{Table $\mathbf{1}$ (on next page)}

The definition of favorable, average and poor prognosis. 
1 Table 1 The definition of favorable, average and poor prognosis.

2

3 prognosis Definition

4 Favorable First embryo transfer and two good-quality embryos transferred A previous successful embryo transfer and $\geq 1$ good-quality embryos transferred

Average First embryo transfer and $\leq 1$ good-quality embryos transferred $\geq 1$ failed embryo transfer and $\geq 1$ good-quality embryos transferred A previous successful embryo transfer and no good-quality embryos transferred Poor $\geq 1$ failed embryo transfer and no good-quality embryos transferred 


\section{Table 2 (on next page)}

Patient characteristics and outcomes of the included cycles with double embryo transfer. 
1 Table 2 Patient characteristics and outcomes of the included cycles with double embryo transfer

\begin{tabular}{|c|c|c|c|c|c|}
\hline \multirow[t]{2}{*}{ Variable } & \multirow{2}{*}{$\begin{array}{l}\text { Included } \\
\text { cycles } \\
(n=2038)\end{array}$} & \multicolumn{3}{|l|}{ Prognosis } & \multirow[t]{2}{*}{$P$-value ${ }^{\mathrm{a}}$} \\
\hline & & $\begin{array}{l}\text { Favorable } \\
(\mathrm{n}=812)\end{array}$ & $\begin{array}{l}\text { Average } \\
(\mathrm{n}=906)\end{array}$ & $\begin{array}{l}\text { Poor } \\
(n=320)\end{array}$ & \\
\hline Maternal age $e^{b, d}$ & $37.8 \pm 2.7$ & $37.6 \pm 2.6$ & $38.0 \pm 2.8$ & $37.9 \pm 2.7$ & 0.007 \\
\hline Maternal BMI & $21.2 \pm 2.5$ & $21.1 \pm 2.5$ & $21.2 \pm 2.5$ & $21.2 \pm 2.4$ & 0.957 \\
\hline \multicolumn{6}{|l|}{ Cause of infertility } \\
\hline Tubal pathology & $682(33.5)$ & $251(30.9)$ & $322(35.5)$ & $109(34.1)$ & 0.124 \\
\hline Endometriosis & $195(9.6)$ & $76(9.5)$ & $89(9.8)$ & $30(9.4)$ & 0.940 \\
\hline Male factor ${ }^{\mathrm{c}, \mathrm{d}}$ & $713(35.0)$ & $265(32.6)$ & $316(34.9)$ & $132(41.3)$ & $<0.001$ \\
\hline Unexplained $^{b, d}$ & $230(11.3)$ & $114(14.0)$ & $90(9.9)$ & $26(8.1)$ & 0.004 \\
\hline Others ${ }^{b, d}$ & $218(10.7)$ & $106(13.1)$ & $89(9.8)$ & $23(7.2)$ & 0.008 \\
\hline Type of infertility (\%) & & & & & 0.069 \\
\hline Primary infertility & $1665(81.7)$ & 647 (79.7) & $760(83.9)$ & $258(80.6)$ & \\
\hline Secondary infertility & $373(18.3)$ & $165(20.3)$ & $146(16.1)$ & $62(19.4)$ & \\
\hline Fertilization method $(\%)^{\mathrm{b}, \mathrm{d}}$ & & & & & 0.006 \\
\hline IVF & $1257(61.7)$ & $534(65.8)$ & $541(59.7)$ & $182(56.9)$ & \\
\hline ICSI & $781(38.3)$ & $278(34.2)$ & $365(40.3)$ & $138(43.1)$ & \\
\hline $\begin{array}{l}\text { Length of embryo } \\
\text { cryopreservation (month) }\end{array}$ & $5.7 \pm 3.8$ & $5.5 \pm 3.9$ & $5.8 \pm 3.7$ & $5.8 \pm 4.1$ & 0.414 \\
\hline Survival rate (\%) & $\begin{array}{l}4119 / 4213 \\
(97.8)\end{array}$ & $\begin{array}{l}1642 / 1681 \\
(97.7)\end{array}$ & $\begin{array}{l}1832 / 1874 \\
(97.8)\end{array}$ & $\begin{array}{r}645 / 658 \\
(98.0)\end{array}$ & 0.879 \\
\hline Damaged blastomere (\%) & & & & & 0.367 \\
\hline Yes & $221(10.8)$ & $80(9.9)$ & $108(11.9)$ & $33(10.3)$ & \\
\hline No & $1817(89.2)$ & $732(90.1)$ & $798(88.1)$ & $287(89.7)$ & \\
\hline Endometrial thickness (mm) & $9.1 \pm 1.6$ & $9.1 \pm 1.6$ & $9.1 \pm 1.6$ & $8.9 \pm 1.7$ & 0.136 \\
\hline Endometrial preparation (\%) & & & & & 0.367 \\
\hline Natural cycles & $126(6.2)$ & $41(5.0)$ & $65(7.2)$ & $20(6.3)$ & \\
\hline HRT cycles & $1912(93.8)$ & $771(95.0)$ & $841(92.8)$ & $300(93.7)$ & \\
\hline $\begin{array}{l}\text { No. of good-quality } \\
\text { embryos transferred }, \text {,c,d }\end{array}$ & $1.1 \pm 0.9$ & $1.9 \pm 0.1$ & $0.8 \pm 0.6$ & 0 & $<0.001$ \\
\hline Clinical pregnancy ${ }^{\mathrm{b}, \mathrm{c}, \mathrm{d}}$ & $816(40.0)$ & $374(46.1)$ & $346(38.2)$ & $96(30.0)$ & $<0.001$ \\
\hline Implantation rate $\mathrm{r}^{\mathrm{b}, \mathrm{c}, \mathrm{d}}$ & $\begin{array}{l}1008 / 4076 \\
(24.7)\end{array}$ & $\begin{array}{l}470 / 1624 \\
(28.9)\end{array}$ & $\begin{array}{l}430 / 1812 \\
(23.7)\end{array}$ & $\begin{array}{l}108 / 640 \\
(16.9)\end{array}$ & $<0.001$ \\
\hline Twin pregnancy & $192(23.5)$ & $\begin{array}{c}102 / 374 \\
(27.3)\end{array}$ & $\begin{array}{l}78 / 346 \\
(22.5)\end{array}$ & $\begin{array}{l}12 / 96 \\
(12.5)\end{array}$ & 0.008 \\
\hline Live birth & $734(36.0)$ & $335(41.3)$ & $318(35.1)$ & $81(25.3)$ & $<0.001$ \\
\hline
\end{tabular}

2 a $P$-value for global comparison between the three groups.

3 b Significant comparison between women with favorable and average prognosis.

$4 \quad$ c Significant comparison between women with average and poor prognosis.

5 d Significant comparison between women with favorable and poor prognosis. 


\section{Table 3 (on next page)}

Clinical outcomes in double embryo transfer, stratified by age and prognosis. 
1 Table 3 Clinical outcomes in double embryo transfer, stratified by age and prognosis.

\begin{tabular}{|c|c|c|c|c|}
\hline & $\begin{array}{l}\text { Favorable } \\
\text { prognosis }\end{array}$ & $\begin{array}{l}\text { Average } \\
\text { prognosis }\end{array}$ & $\begin{array}{l}\text { Poor } \\
\text { prognosis }\end{array}$ & $P$-value ${ }^{\mathrm{a}}$ \\
\hline \multicolumn{5}{|l|}{$35-37 \mathrm{y}$} \\
\hline No. of cycles & 487 & 483 & 164 & \\
\hline No. of pregnancies & 240 & 198 & 59 & \\
\hline Clinical pregnancy rate $\mathrm{e}^{\mathrm{b}, \mathrm{d}}$ & $49.3 \% \mathrm{e}^{\mathrm{e}}$ & $41.0 \% \mathrm{e}^{\mathrm{e}}$ & $36.0 \% \mathrm{e}^{\mathrm{e}}$ & 0.003 \\
\hline Implantation rate $e^{b, d}$ & $310 / 974(31.8)$ & $249 / 966(25.8)$ & $68 / 328(20.7)$ & $<0.001$ \\
\hline No. of twin pregnancies & 70 & 51 & 9 & \\
\hline Twin pregnancy rate & $29.2 \%$ & $25.8 \%$ & $15.3 \%$ & 0.092 \\
\hline Live birth rate $\mathrm{r}^{\mathrm{b}, \mathrm{d}}$ & $217(44.6)^{\mathrm{g}, \mathrm{i}}$ & $185(38.3)^{\mathrm{g}}$ & $51(31.1)^{\mathrm{g}}$ & 0.006 \\
\hline \multicolumn{5}{|l|}{$38-40 y$} \\
\hline No. of cycles & 221 & 262 & 98 & \\
\hline No. of pregnancies & 97 & 100 & 29 & \\
\hline Clinical pregnancy rate & $43.9 \%$ & $38.2 \%$ & $29.6 \%{ }^{\mathrm{f}}$ & 0.051 \\
\hline Implantation rate red $^{\mathrm{c} d}$ & $123 / 442(27.8)$ & $122 / 524(23.3)$ & $32 / 196(16.3)$ & 0.007 \\
\hline No. of twin pregnancies & 26 & 22 & 3 & \\
\hline Twin pregnancy rate & $26.8 \%$ & $22.0 \%$ & $10.3 \%$ & 0.173 \\
\hline Live birth rate & $86(38.9)$ & $91(34.7)$ & $24(24.5)^{\mathrm{h}}$ & 0.044 \\
\hline \multicolumn{5}{|l|}{$>40 \mathrm{y}$} \\
\hline No. of cycles & 104 & 161 & 58 & \\
\hline No. of pregnancies & 37 & 48 & 8 & \\
\hline Clinical pregnancy rate ${ }^{c, d}$ & $35.6 \%$ & $29.8 \%$ & $13.8 \%$ & 0.012 \\
\hline Implantation rate $\mathrm{c}^{\mathrm{c}, \mathrm{d}}$ & $43 / 208(20.7)$ & $53 / 322(16.5)$ & $8 / 116(6.9)$ & 0.039 \\
\hline No. of twin pregnancies & 6 & 5 & 0 & \\
\hline Twin pregnancy rate & $16.2 \%$ & $10.4 \%$ & $0 \%$ & 0.553 \\
\hline Live birth rate $e^{c, d}$ & $32(30.8)$ & $42(26.1)$ & $6(10.3)$ & 0.013 \\
\hline $\begin{array}{l}P \text { value associated with the age } \\
\text { and clinical pregnancy rate in } \\
\text { each prognostic category }\end{array}$ & 0.031 & 0.041 & 0.007 & \\
\hline $\begin{array}{l}P \text { value associated with the age } \\
\text { and twin pregnancy rate in } \\
\text { each prognostic category }\end{array}$ & 0.256 & 0.076 & 0.708 & \\
\hline $\begin{array}{l}P \text { value associated with the age } \\
\text { and live birth rate in each } \\
\text { prognostic category }\end{array}$ & 0.025 & 0.019 & 0.007 & \\
\hline \multicolumn{5}{|c|}{ a $P$-value for global comparison between the three groups. } \\
\hline
\end{tabular}


7 fignificant comparison in clinical pregnancy rate between women aged 38-40 years and $>40$ years.

$8{ }^{\mathrm{g}}$ Significant comparison in live birth rate between women aged 35-37 years and $>40$ years.

$9{ }^{\mathrm{h}}$ Significant comparison in live birth rate between women aged $38-40$ years and $>40$ years.

10 i Significant comparison in live birth rate between women aged 35-37 years and 38-40 years.

11 